Published by Avanti Publishers

Global Journal of Earth

Science and Engineering

ISSN (online): 2409-5710

\title{
Application of Improved Fuzzy-Set Pair Analysis in Mine Water Environmental Quality Evaluation
}

\author{
Shi Longqing, He Li and Zhai Peihe* \\ School of Earth Science and Engineering, Shandong University of Science and Technology, Qingdao, Shandong 266590, China
}

\section{ARTICLE INFO}

Article Type: Research Article

Keywords:

Fuzzy -SPA method

Grey clustering method

Water quality evaluation

The mine water resources

Improvement in Nemerow index method

Timeline:

Received: August 16, 2021

Accepted: September 16, 2021

Published: October 06, 2021

Citation: Longqing S, Li H, Peihe Z. Application of Improved Fuzzy-Set Pair Analysis in Mine Water Environmental Quality Evaluation. Glob J Earth Sci Eng. 2021; 8: 35-44.

DOI: https://doi.org/10.15377/2409-5710.2021.08.3

\begin{abstract}
Mine water is an important water resource. The utilization of mine water resources is of great significance to the realization of sustainable economic and social development, and water quality evaluation is an important part of mine water resources utilization. In this paper, the improved Nemerow index method is applied to the compound algorithm of the fuzzy-set pair analysis method, and the water quality evaluation of the Kongzhuang Mine in Xuzhou is carried out. Finally, compared with the evaluation results of the set pair analysis method and grey clustering method, it is shown that the evaluation results of this method are reasonable and scientific, which can provide a reliable basis for the utilization of mine water resources.
\end{abstract}

*Corresponding Author Email:13953891430@163.com Tel: 13953891430

(C2021 Longqing et al. Published by Avanti Publishers. This is an open access article licensed under the terms of the Creative Commons Attribution Non-Commercial License which permits unrestricted, non-commercial use, distribution and reproduction in any medium, provided the work is properly cited. (http://creativecommons.org/licenses/by-nc/4.0/) 


\section{Introduction}

China is the country with the largest amount of coal mining in the world. At the same time, the coal mine water emissions and production are also among the top of the world [1]. According to the statistics of the China National Coal Industry Association, the comprehensive utilization rate of mine water in China was 75.8\% in 2019. However, due to the influence of various factors such as water quality and quantity, the utilization rate of mine water was different, and the utilization rate of some mine water was still low. The inefficient utilization of mine water not only leads to a large amount of waste of water resources but also leads to the imbalance of the ecological environment [2]. Therefore, a comprehensive water quality evaluation must be carried out to meet the requirements of mine water discharge and production and living use [3].

At present, there are many methods for water quality evaluation, including the neural network method $[4,5]$, single-factor index method [6], fuzzy comprehensive evaluation method [7,8], and grey clustering method $[9,10]$. These methods have certain deficiencies in water quality evaluation. For example, the neural network learning data is big, has slow convergence speed, and is easy to fall into local minimum; The single factor index method is simple in the calculation but conservation in evaluation results; The fuzzy comprehensive evaluation method is complex in calculation and has strong subjectivity in determining index weight vector; The grey clustering method has strong subjectivity and a huge amount of calculation [11]. The selection of water quality evaluation indexes has the characteristic of fuzziness, randomness, and uncertainty [12]. Water quality evaluation is essentially a process of analyzing the deterministic evaluation criteria and the uncertain monitoring data [13]. Set pair analysis treats the problem of deterministic and uncertain as an uncertain system, which can comprehensively reflect the random, fuzzy, gray, and other common uncertain factors in the water quality system. The traditional fuzzy theory has some limitations, such as loss of index information and the repeated calculation of index concentration. Accordingly, the deficiency is compensated by improving the coincidence algorithm of fuzzy theory. The two theories are combined to establish an improved fuzzy-set pair analysis water quality evaluation model to evaluate the quality of mine water, which provides a new method for the scientific evaluation of groundwater environmental quality.

\section{Materials and Methods}

\subsection{The Study Area}

The Kongzhuang coal mine in Xuzhou is located in Peixian County, Jiangsu province, and Weishan County, Shandong Province, $80 \mathrm{~km}$ away from Xuzhou city. The geographical coordinates of the main shaft are $116^{\circ} 57^{\prime} 13$ "E, 34 50 " 20 "N. The total area of the mine is about $44.13 \mathrm{~km}^{2}$. The mining area has convenient transportation, and there is a special line for Xu Pei Railway. The minefield landform belongs to the Huang-Huai-Hai alluvial plain, flat terrain, high east, low west terrain. This area is a temperate semi-humid monsoon climate with high temperatures and heavy rainfall in summer. The annual average precipitation is $738.2 \mathrm{~mm}$, and the annual average temperature is $14.2^{\circ} \mathrm{C}$. The main water-filling aquifers in the Kongzhuang well field are the bottom aquifer of quaternary, the sandstone in the roof of the coal seam, and the four ash aquifers. Faults dominate the structure in this area, and faults are more developed.

\subsection{The Data Source and Evaluation Grade Standard}

The water quality samples in this paper are from No.7 and No.8 coals in Xuzhou Kongzhuang Mine, Xuzhou [14], among which eight water quality samples were collected from No.7 coal and six water quality samples from No.8 coal. The measured date of sulfate, chloride, total hardness, and salinity are selected as the main evaluation indexes, as shown in Table 1. In this paper, 《Groundwater Environmental Quality Standard》 (GB/T 14848-2017) was adopted as the basis for water quality and environmental assessment [15]. Groundwater quality levels are shown in Table 2. 
Table 1: Mine water of the monitoring of water quality monitoring results.

\begin{tabular}{|c|c|c|c|c|c|}
\hline \multirow{2}{*}{ Monitoring Stations } & \multicolumn{5}{|c|}{$\mathrm{u} / \mathrm{mg} \cdot \mathrm{L}^{-1}$} \\
\hline & The Sample Source & Sulfate & Chloride & Total Hardness & Mineralization \\
\hline S7-1 & 7 coal roof water & 1029 & 212.9 & 1044.13 & 2048.46 \\
\hline $\mathrm{S} 7-2$ & 7 coal roof water & 67.09 & 73.17 & 196.51 & 597.86 \\
\hline S7-3 & 7 coal roof sandstone & 303.64 & 83.64 & 36.87 & 1276.35 \\
\hline S7-4 & 7 coal roof sandstone & 341.42 & 92.99 & 40.68 & 1415.24 \\
\hline S7-5 & 7 coal roof water & 267.09 & 208.29 & 31.1 & 1526.64 \\
\hline S7-6 & 7 coal roof sandstone & 1006.53 & 289.33 & 524.78 & 2242.43 \\
\hline S7-7 & 7 coal roof sandstone & 1474.56 & 268.31 & 664.77 & 2829.9 \\
\hline S7-8 & 7 coal roof water & 1223.28 & 264.8 & 1073.64 & 2416.28 \\
\hline S8-1 & 8 coal roof water & 1353.06 & 269.33 & 1295.42 & 2577.86 \\
\hline S8-2 & 8 coal roof water & 1075.31 & 256.72 & 1116.54 & 2177.31 \\
\hline S8-3 & 8 coal roof water & 1425.78 & 271.42 & 1277.54 & 2709.91 \\
\hline S8-4 & 8 coal roof sandstone & 1313 & 268.83 & 1208.65 & 2542.75 \\
\hline S8-5 & 8 coal roof water & 627.69 & 253.36 & 95.18 & 1835.28 \\
\hline S8-6 & 8 coal roof water & 1317.12 & 266.22 & 1158.45 & 2553.79 \\
\hline
\end{tabular}

Table 2: Groundwater classification standard.

\begin{tabular}{|c|c|c|c|c|}
\hline The Water Quality Level & Sulfate & Chloride & Total Hardness & Mineralization \\
\hline I & $\leq 50$ & $\leq 50$ & $\leq 150$ & $\leq 300$ \\
\hline II & $\leq 150$ & $\leq 150$ & $\leq 300$ & $\leq 500$ \\
\hline III & $\leq 250$ & $\leq 250$ & $\leq 450$ & $\leq 1000$ \\
\hline IV & $\leq 350$ & $\leq 350$ & $\leq 650$ & $\leq 2000$ \\
\hline $\mathrm{V}$ & $>350$ & $>350$ & $>650$ & $>2000$ \\
\hline
\end{tabular}

\subsection{Methods}

\subsubsection{SPA}

Set pair analysis (SPA) [16], also known as relation mathematics, is a new systematic analysis method that uses relation degree to deal with uncertain problems, proposed by Zhao Keqin, a Chinese scholar. Its core idea is to analyze and deal with the deterministic relation and the uncertain relation between the objective things to be studied as an uncertain system [17], and this method analyzes the things and their systems through the three aspects of identity, difference, and opposition. In an uncertain system, the two related sets $X$ and $Y$, both of which have characteristics of $N$ terms, constitute a set pair $H(X, Y)$. The properties of sets $X$ and $Y$ are treated as a system, and the identity, differential form, and antithesis of sets $X$ and $Y$ in set pairs are analyzed. According to the relationship among the same, different, and opposite, the correlation degree can be expressed as:

$$
\mu(H)=\frac{S}{N}+\frac{F}{N} i+\frac{P}{N} j=a+b i+c j(a+b+c=1)
$$


In this formula, $\mu(H)$ is the connection degree of the set to $H(X, Y) ; N$ is the total number of elements in the set, $N=S+P+F$; $S$ is the number of elements in the same condition of two sets; $P$ for two set in the opposite state the number of elements; $F=N-S-P$ for two sets the number of elements in a different state; $i$ is the difference coefficient and $i \in(-1,1) ; j$ is the coefficient of antithesis identification, and its value is defined as -1 . Order $a=\frac{S}{N}, b=\frac{F}{N}$, and $c=\frac{P}{N}$ represent the identity, difference, and opposition of the set, respectively.

The groundwater environmental quality can be divided into five grades: I, II, II IV, V, which are used as evaluation criteria respectively, $Y_{5}=\{\mathrm{I}, \mathrm{II}, \mathrm{III}, \mathrm{IV}, \mathrm{V}\}$. The $n$ evaluation indexes selected from the monitoring points are denoted as $X_{n}$, from which the set pair $H\left(X_{n}, Y_{5}\right)$ is obtained. According to the definition of the set pair analysis, class I with class V are regarded as the same degree, and $\mathbb{I}, \mathbf{I I}, \mathrm{IV}$ are regarded as the different degree, and the five-element connection degree is established:

$$
\mu(H)=a+b i+c j+d k+e l
$$

Among them, $a 、 b 、 c 、 d$ and e represent the connection degree between an evaluation index and water quality standard and meet the normalization condition. $i 、 j 、 k 、 l$ are only used as markers.

Since all the indexes selected in this article are of the smaller type, the better type, the calculation formula of the connection degree is as follows:

$$
\mu_{j k}=\left\{\begin{array}{c}
\frac{S_{1-x}}{S_{1}-0}+\frac{x-0}{s_{1}-0}+0_{i}+0_{j}+0_{k}+0_{l} x \in\left[0, S_{1}\right] \\
\frac{S_{2-} x}{S_{2-} S_{1}}+\frac{x-S_{1}}{S_{2-} S_{1}} i+0_{j}+0_{k}+0_{l} x \in\left(S_{1}, S_{2}\right] \\
0+\frac{S_{3-} x}{S_{3-} S_{2}} i+\frac{x-S_{2}}{S_{3-} S_{2}} j+0_{k}+0_{l} x \in\left(S_{2}, S_{3}\right] \\
0+0_{i}+\frac{S_{4-} x}{S_{4-} S_{3}} j+\frac{x-S_{3}}{S_{4-} S_{3}} k+0_{l} x \in\left(S_{3}, S_{4}\right] \\
0+0_{i}+0_{j}+0_{k}+1_{l} x \in\left(S_{4},+\infty\right)
\end{array}\right.
$$

In the formula, $\mu_{j k}$ is the connection degree of the $k$ index of the monitoring point $j ; S_{1} 、 S_{2} 、 S_{3} 、 S_{4}$ are the threshold of the standards of grade I, II, II, IV of the water quality grade respectively; $x$ as individual monitoring points of index values. $n$ indicators of each monitoring point and their corresponding $p$ levels of connection number constitute the connection number matrix $Q$ :

\subsubsection{Determine Index Weight}

$$
Q=\left[\begin{array}{cccc}
\mu_{11} & \mu_{12} & \cdots & \mu_{1 p} \\
\mu_{21} & \mu_{22} & \cdots & \mu_{2 p} \\
\vdots & \vdots & \\
\mu_{n 1} & \mu_{n 2} & \cdots & \mu_{n p}
\end{array}\right]
$$

In the comprehensive evaluation of groundwater environment quality, it is necessary to consider the difference in the contribution of each indicator to groundwater environmental quality and determine the weight of each indicator. In the comprehensive water quality evaluation, the weight method can be divided into two categories: subjective weight method and objective weight method [18]. Experts determine subjective weighting evaluation according to their experience, which is highly subjective and arbitrary. The objective weighting method reflects the utility value of the data itself, which can effectively reduce the advantages of subjectivity and reduce the influence of human factors. Therefore, this paper selects the objective weighting method combining the entropy weighting method and the overbid multiple weighting method to determine the weights of each evaluation index to make the evaluation results more objective and practical.

\section{(1) Entropy Weight Method}

The entropy weight method is an objective weighting method. The smaller the information entropy of an index is, the greater the variation degree of the index will be, the more information will be provided, and the higher the 
corresponding weight will be [19]. The calculation steps of the entropy weight method are as follows:

Step 1: Build the evaluation index system

There are $m$ evaluation samples in the water quality evaluation, and each sample has $n$ evaluation indexes. The original matrix $R$ of the data is stablished:

$$
R=\left(r_{i j}\right) m \times n, \quad(i=1,2,3, \ldots, m ; j=1,2,3, \ldots, n)
$$

$r_{i j}$ is the measured value of evaluating sample $i$ under index $j$.

Step 2: Data normalization processing

Due to the different dimensions of different indexes, there are great differences in the order of magnitude. In order to eliminate the influence of dimensions and order of magnitude among different indexes, the normalized processing of index data is carried out to get the normalized judgment matrix $R^{\prime}$ :

$$
\begin{gathered}
a_{i j}=\frac{r_{i j}-r_{\min }}{r_{\max }-r_{\min }} \\
R^{\prime}=\left\{\begin{array}{c}
a_{11} \ldots a_{1 n} \\
\vdots \vdots \\
a_{m 1} \ldots a_{m n}
\end{array}\right\}
\end{gathered}
$$

Step 3: Determine the entropy value $H_{j}$ of the j-th evaluation index :

$$
\left\{\begin{array}{c}
H_{j}=-\frac{1}{m n} \sum_{i=1}^{m}\left(p_{i j} \ln p_{i j}\right) \\
p_{i j}=\frac{a_{i j}}{\sum_{i=1}^{m} a_{i j}}
\end{array}\right.
$$

When $p_{i j}=0, \ln p_{i j}$ is meaningless. So the improvement of information entropy is as follows:

$$
p_{i j}=\frac{1+a_{i j}}{\sum_{i=1}^{m} 1+a_{i j}}
$$

Step 4: Calculate the weight of the $j$ evaluation item:

$$
W_{j}^{\prime}=\left(1-H_{j}\right) /\left(n-\sum_{j=1}^{n} H_{j}\right),\left(\sum_{j=1}^{n} W_{j}=1\right)
$$

\section{(2) Excessive Ratio Method}

The excessive ratio method can not only highlight the role of the main pollutants in the groundwater environmental quality evaluation but also consider the difference in standard values of different indexes [20]. Its calculation formula is as follows:

$$
W_{j}^{\prime \prime}=\sum_{i=1}^{m} \frac{r_{i j}}{\overline{s_{J}}} / \sum_{j=1}^{n} \sum_{i=1}^{m} \frac{r_{i j}}{\overline{s_{J}}}
$$

$\overline{s_{J}}$ is the arithmetic mean value of the four evaluation grade thresholds from I to IV of the $j$ evaluation index.

\section{(3) The Comprehensive Weights}

By combining the advantages of the entropy weight method and the excessive ratio weight method, the 
following comprehensive weights are obtained, and the weights meet the normalization:

$$
\mathrm{W}=\frac{W_{J}^{\prime} W_{J}^{\prime \prime}}{\sum_{j=1}^{n}\left(W_{J}^{\prime} W_{J}^{\prime \prime}\right)}
$$

\subsubsection{The Theory of Fuzzy Composite Algorithm}

Fuzzy comprehensive evaluation synthesis algorithm has some shortcomings, such as repeated calculation of index concentration, information loss, and weight effect is not obvious, so this paper uses improved Nemerow algorithm to overcome these defects.

The traditional Nemerow index method overemphasizes the influence of the maximum weight index on the water quality grade [21]. In order to comprehensively consider the weight of each evaluation index in the water quality evaluation and dilute the influence of the maximum weight index, the Nemerow algorithm is improved. The calculation formula is as follows:

$$
\begin{gathered}
P=\sqrt{\left(\bar{F}_{\text {max }}^{2}+\bar{F}_{\mathrm{i}}^{2}\right)} \\
F_{i}=\frac{c_{i}}{s_{i j}} i=1,2, \cdots n, j=1,2, \cdots, m
\end{gathered}
$$

$c_{i}$ is the measured value of the evaluation index of class $i, s_{i j}$ is the standard value of class $j$ of the evaluation index of class $i, \bar{F}_{\text {max }}^{2}$ is the average value of $F_{i}$ and $F_{\text {max }}$ corresponds to the index with the largest weight, and $P$ is the improved Nemerow index.

\subsubsection{The Algorithm of Improved Fuzzy-Set Pair Analysis}

The improved algorithm of fuzzy - set pair analysis formula is as follows:

$$
C=W \oplus Q=\left(w_{1}, w_{2} \cdots w_{n}\right) \oplus\left(\begin{array}{cccc}
\mu_{11} & \mu_{12} & \cdots & \mu_{1 p} \\
\mu_{21} & \mu_{22} & \cdots & \mu_{2 p} \\
\vdots & \vdots \\
\mu_{n 1} \mu_{n 2} & \cdots & \mu_{n p}
\end{array}\right)=\left(\begin{array}{cccc}
F_{11} F_{12} & \cdots & F_{1 p} \\
F_{21} & F_{22} & \cdots & F_{2 p} \\
\vdots & \vdots \\
F_{n 1} & F_{n 2} & \cdots & F_{n p}
\end{array}\right)
$$

$\oplus$ is the improved composition operator. According to the improved Nemerow index algorithm:

$$
C=\left(f_{1}, f_{2}, \cdots, f_{p}\right)=\left(\sqrt{F_{1 \text { max }}^{2}+\bar{F}_{1}^{2}}, \sqrt{F_{2 \text { max }}^{2}+\bar{F}_{2}^{2}}, \cdots, \sqrt{F_{\text {pmax }}^{2}+\bar{F}_{P}^{2}}\right)
$$

$F_{i \text { max }}$ represents the product of the maximum index of weight and the average value of the maximum value of $F_{1 i}, F_{2 i}, \cdots, F_{n i}, \bar{F}_{l}$ is the average value of $F_{1 i}, F_{2 i}, \cdots, F_{n i}, i=1,2, \cdots, p$.

\subsubsection{Judging Water Quality Grade}

Judge the water quality grade of the monitoring point according to the principle of maximum membership:

$$
f_{j}=\max \left(f_{1}, f_{2}, \cdots, f_{p}\right)
$$

\section{Results and Discussion}

\subsection{Result}

Taking the monitoring point S7-1 as an example, the correlation degree of each index can be obtained according to formula (3):

$$
\mu_{1 \text { sulfate }}=0+0_{i}+0_{j}+0_{k}+1_{l}
$$




$$
\begin{gathered}
\mu_{1 \text { chloride }}=0+\frac{250-212.9}{250-150} i+\frac{212.9-150}{250-150} j+0_{k}+0_{l}=0+0.371 i+0.629 j+0_{k}+0_{l} \\
\mu_{1 t} \quad \text { hardness } \\
=0+0_{i}+0_{j}+0_{k}+1_{l} \\
\mu_{1 \text { mineralizatio }}=0+0_{i}+0_{j}+0_{k}+1_{l}
\end{gathered}
$$

Therefore, the various indicators monitoring point S7-1 contact degree matrix can be expressed as:

$$
\left[\begin{array}{c}
0,0,0,0,1 \\
0,0.371,0.629,0,0 \\
0,0,0,0,1 \\
0,0,0,0,1
\end{array}\right]
$$

According to formula (5) (12), the index weight is obtained, as shown in Table 3:

Table 3: The evaluation index weight.

\begin{tabular}{|c|c|c|c|c|}
\hline Index & Sulfate & Chloride & Total Hardness & Mineralization \\
\hline \hline weight & 0.483 & 0.119 & 0.165 & 0.223 \\
\hline
\end{tabular}

The comprehensive connection degree of monitoring point S7-1 can be calculated by the formula (13) (15) :

$$
C=\left(\begin{array}{llll}
0, & 0.2465 & 0.0419, \quad 0, & 0.5308
\end{array}\right)
$$

\begin{tabular}{|c|c|c|c|c|c|}
\hline \multirow{2}{*}{ Monitoring Point } & \multicolumn{5}{|c|}{ Comprehensive Connection Degree of Each Level } \\
\hline & I & II & III & IV & $\mathrm{V}$ \\
\hline S7-1 & 0 & 0.2465 & 0.0419 & 0 & 0.5308 \\
\hline S7-2 & 0.4284 & 0.1607 & 0.0255 & 0 & 0 \\
\hline S7-3 & 0.1026 & 0.0224 & 0.2447 & 0.4782 & 0 \\
\hline S7-4 & 0 & 0.1010 & 0.0454 & 0.1670 & 0.0541 \\
\hline S7-5 & 0.0922 & 0.0277 & 0.4259 & 0.1147 & 0 \\
\hline S7-6 & 0 & 0 & 0.0678 & 0.0359 & 0.9990 \\
\hline S7-7 & 0 & 0 & 0.0542 & 0.0227 & 0.5308 \\
\hline $57-8$ & 0 & 0 & 0.0565 & 0.0101 & 0.5308 \\
\hline S8-1 & 0 & 0 & 0.0537 & 0.0129 & 0.5308 \\
\hline S8-2 & 0 & 0 & 0.0621 & 0.0045 & 0.5308 \\
\hline S8-3 & 0 & 0 & 0.0525 & 0.0258 & 0.5308 \\
\hline S8-4 & 0 & 0 & 0.0542 & 0.0123 & 0.5308 \\
\hline S8-5 & 0.0922 & 0 & 0.0691 & 0.1095 & 0.4979 \\
\hline S8-6 & 0 & 0 & 0.0559 & 0.1090 & 0.5308 \\
\hline
\end{tabular}

According to the principle of maximum membership, the water quality grade of monitoring point S7-1 is $v$.

Similarly, the comprehensive connection degree of other monitoring points can be calculated, as shown in Table 4:

Table 4: Comprehensive connection degree of each monitoring point. 
According to the principle of the maximum degree of membership, the water quality level of each monitoring point can be judged. In order to verify the effectiveness of the improved fuzzy-set pair analysis method, the results of the set pair analysis and gray cluster analysis are compared, as shown in Table $\mathbf{5}$.

\subsection{Discuss}

(1) In this paper, the improved fuzzy-set pair analysis method is used to analyze the mine water quality environment of Xuzhou Kongzhuang Coal Mine. The results are generally consistent with the evaluation results of the set pair analysis method and the grey clustering evaluation method, and the coincidence degree reaches $79.6 \%$, which fully indicates that the evaluation method can be applied in the groundwater evaluation. According to Table 5, The evaluation results of the 14 monitoring points were consistent except for the monitoring points S73, S7-4, and 58-5. For the monitoring points S7-3 and S7-4, two indexes are above grade II, and one index is between grades I and II. Considering the influence of the weight of each evaluation index, it is more rigorous and scientific to evaluate it as grade IV.

(2) As can be seen from Table 5, among the 14 monitoring points in Xuzhou Kongzhuang coal Mine, only the S72 monitoring point has grade I water quality, 2 of the well water samples from coal mine 7 are grade IV, 1 is grade $\mathrm{II}$, and 4 are grade $\mathrm{V}$. The water quality of 6 monitoring points in coal mine 8 is all grade $\mathrm{V}$, and the water quality of coal mine 7 is generally better than that of coal mine 8 .

Table 5: Evaluation results and comparison.

\begin{tabular}{|c|c|c|c|}
\hline Monitoring Point & Improved F-SPA & SPA & Grey Clustering Method \\
\hline S7-1 & $\mathrm{V}$ & $\mathrm{V}$ & $\mathrm{V}$ \\
\hline S7-2 & I & I & I \\
\hline S7-3 & IV & III & I \\
\hline S7-4 & IV & III & I \\
\hline S7-5 & III & III & III \\
\hline S7-6 & $\mathrm{V}$ & $\mathrm{V}$ & $\mathrm{V}$ \\
\hline S7-7 & $\mathrm{V}$ & $\mathrm{V}$ & $\mathrm{V}$ \\
\hline S7-8 & $\mathrm{V}$ & $\mathrm{V}$ & $\mathrm{V}$ \\
\hline S8-1 & $\mathrm{V}$ & V & $\mathrm{V}$ \\
\hline S8-2 & $\mathrm{V}$ & $\mathrm{V}$ & $\mathrm{V}$ \\
\hline S8-3 & $\mathrm{V}$ & $\mathrm{V}$ & $\mathrm{V}$ \\
\hline S8-4 & $\mathrm{V}$ & $\mathrm{V}$ & $\mathrm{V}$ \\
\hline S8-5 & $\mathrm{V}$ & IV & III \\
\hline S8-6 & $\mathrm{V}$ & $\mathrm{V}$ & $\mathrm{V}$ \\
\hline
\end{tabular}

(3) According to the measured values and evaluation results of the monitoring point indexes, the salinity content of all the monitoring points with grade $v$ inferior water is basically greater than $2000 \mathrm{mg} / \mathrm{L}$, among which sulfate has the largest contribution rate to salinity. Biological desulfurization and permeability reflection wall methods can be used to reduce the sulfate in mine water.

\section{Conclusion}

In this paper, an improved fuzzy-set pair analysis model is constructed by combining the matching algorithm of improved fuzzy theory with set pair analysis, and the mine water quality of 12 monitoring points in the Xuzhou Kongzhuang coal mine is evaluated. The results show that the model is scientific and reasonable. 
(1) The entropy-multiple weighting method is relatively more objective, which overcomes the interference of human factors caused by the simple subjective weighting method and makes the evaluation results closer to the actual situation.

(2) The improved fuzzy-set pair analysis method makes comprehensive use of the evaluation information of the connection degree between the measured value of the monitoring point evaluation index and the groundwater environmental standard and uses the improved fuzzy theory to accord with the algorithm to highlight the role of the weight, avoids the loss of information, and the evaluation results are comparatively more objective. The results generally agree with the evaluation results of set pair analysis and grey clustering method, indicating that this method is suitable for mine water quality evaluation.

(3) The water quality of the Xuzhou Kongzhuang coal mine is generally poor, so corresponding measures should be taken to improve the utilization efficiency of mine water and the ecological environment of the mining area.

\section{Acknowledgment}

The authors thank all the experts for their careful work and thoughtful suggestions. We gratefully acknowledge the financial support of the Key Projects of Natural Science Foundation of Shandong Province (ZR2020KE023) and the National Natural Science Foundation of China (42002282, 51804184, 41807283).

\section{References}

[1] Liu LM, Lian CJ, et al. Underground mine water disposal method and water supply system [j]. Coal Engineering. 2003; (09): 58-60.

[2] Wang HD. Treatment and Utilization of Mine Water [j]. Energy and Energy Conservation. 2011; (03): 74-75.

[3] Mao WD, Zhou RL, et al. Zero liquid discharge treatment technology and application for coal mine drainage water [j]. Coal Science and Technology, 2017, 45(11): 205-210.

[4] Du SS, Wang HQ, et al. Improved Modal Model of Groundwater Quality Evaluation Based on Artificial Neural Network [j]. Journal of Beijing Normal University(Natural Science), 2014; 50(4): 424-428.

[5] Yue DD, Liang HT, et al. Application of BP artificial neural network model in groundwater quality evaluation of west Anshan Iron Mine [j]. The Administration and Technique of Environmental Monitoring, 2016; 28(4): 23-24.

[6] Xie WP, Yang L, et al. Application and Analysis of Different Water Quality Evaluation Methods for the Caoqiao River [j]. The Administration and Technique of Environmental Monitoring, 2013; 25(02): 62-66.

[7] Ws S, Xa Q, et al. Groundwater quality Classification for Abandoned Mine Based on Fuzzy Comprehensive Evaluation [j]. China Mining Magazine, 2021; 30(02): 167-171.

[8] Li XL, Wang LG, et al. Improvement and Application of Fuzzy Comprehensive Evaluation Method for Mine Water Quality [j]. Journal of Water Resources and Water Engineering, 2009; 20(05): 5-8.

[9] Dong Y, Li J, et al. Application of Grey Clustering Method Based on Level Eigenvalue in Water Quality Evaluation of Yellow River [J]. Environmental Science and Management, 2019; 44(10): 175-179.

[10] Yin SS, Zhu YM. Comprehensive Assessment of Water Quality by Using Gray-clustering Law and Artificial Neural Network [J]. Journal of Green Science and Technology, 2014; (03): 75-78.

[11] Zhang YB, Si XL. Quality Assessment of Surface Water Environment Based on Improved Grey Correlation Metho [J]. Yellow River, 2017; 39(11): 109-111+127.

[12] Gang ST, Jia T, et al. Assessment of Groundwater Quality in Hamatong Drainage Basin by Using Model of Set Pair Analysis Based on Entropy Weight Method [j]. Journal of Yangtze River Scientific Research Institute, 2018; 35(9): 23-27.

[13] Meng CC. Comprehensive Assessment on the Water Environment Quality of Surface-Based on Set Pair Analysis-Variable Fuzzy Set Model [j]. Journal of Anhui Agricultural Science, 2010; 38(22): 11897-11899.

[14] Li T. Application of grey clustering method in a comprehensive evaluation of mine water quality [j]. Light Industry Science and Technology, 2017; (11): 100-101.

[15] GB/T 14848-2017, The Groundwater Quality Standard [S].

[16] Li KQ. Set Pair Analysis and Its Preliminary Application [j]. Discovery of Nature, 1994; (01): 67-72.

[17] Liu Q, Wang M, et al. The application of set pair analysis in groundwater quality evaluation was analyzed [J]. China Standardization, 2019; (20): 107-108. 
[18] Wu DJ, Wang JS, Ding AZ. Comparison of Two Methods of Determining Index Weight During Groundwater Quality Assessment [j]. Geotechnical Investigation and Surveying, 2006; (07): 17-22+34+31.

[19] Lai ZW, Mao ZY, et al. Water Quality Evaluation Method Based on Entropy Weight-Partial Order Set [j]. Journal of Yangtze River Scientific Research Institute, 2021; 38(03): 32-38.

[20] Hu QJ. Application of attribute recognition model based on superstandard multiple method in surface water quality evaluation [j]. Jiangsu Water Resources, 2021; (05): 31-36.

[21] Li S, Xu D, et al. Application of Improved Nemerow Index Method in Reservoir Water Quality Evaluation [j]. Science Technology and Engineering, 2020; 20(31): 13079-13084. 